\title{
Response of an aggressive periosteal aneurysmal bone cyst $(A B C)$ of the radius to denosumab therapy
}

\author{
Chantal Pauli ${ }^{1}$, Bruno Fuchs ${ }^{2}$, Christian Pfirrmann ${ }^{3}$, Julia A Bridge ${ }^{4}$, Silvia Hofer ${ }^{5}$ and Beata Bode ${ }^{\text {** }}$
}

\begin{abstract}
Aneurysmal bone cyst (ABC), once considered a reactive lesion, has been proven to be a neoplasia characterized by rearrangements of the USP6-gene. Aggressive local growth and recurrences are common and therapeutic options may be limited due to the vicinity of crucial structures. We describe a case of a locally aggressive, multinucleated giant cell-containing lesion of the forearm of a 21-year old woman, treated with denosumab for recurrent, surgically uncontrollable disease. Under the influence of this RANKL inhibitor, the tumor showed a marked reduction of the content of the osteoclastic giant cells and an extensive metaplastic osteoid production leading to the bony containment, mostly located intracortically in the proximal radius. The diagnosis of a periosteal ABC was confirmed by FISH demonstrating USP6 gene rearrangement on the initial biopsy. Function conserving surgery could be performed, enabling reconstruction of the affected bone. Inhibition of RANKL with denosumab may offer therapeutic option for patients not only with giant cell tumors but also with ABCs.
\end{abstract}

Keywords: Aneurysmal bone cyst, FISH, USP6, Denosumab

\section{Background}

Aneurysmal bone cysts (ABCs) are rare benign skeletal tumors usually occurring in the first two decades of life and typically associated with a growing mass, swelling, pain and bone destruction [1,2]. Approximately $70 \%$ of the cases are considered primary lesions and the remaining 30\% arising secondary to different primary tumors such as osteoblastoma, giant cell tumor of bone, chondroblastoma, fibrous dysplasia or low grade intramedullary osteosarcoma [3]. It has been shown that primary $\mathrm{ABCs}$, initially considered to be reactive and non-neoplastic $[1,4]$, correspond to a neoplastic disorder associated with a specific set of genetic aberrations $[5,6]$. Over the past few years many different translocations have been described in $\mathrm{ABCs}$, all resulting in oncogenic activation of the USP6 gene localized to $17 \mathrm{p} 13$ [7-9].

Primary ABCs can affect any bone. Usually they arise in the metaphysis of long bones such as the femur, tibia

\footnotetext{
* Correspondence: beata.bode@usz.ch

${ }^{1}$ Institute of Surgical Pathology, University Hospital Zurich, $\mathrm{CH}-8091$, Zurich, Switzerland

Full list of author information is available at the end of the article
}

and humerus; however, the spine may also be affected with compression/infiltration of nerves and spinal cord causing neurological symptoms [10]. The magnetic resonance imaging (MRI) demonstrates an intraosseous, expansile, lytic, eccentric, septated lesion, containing characteristic fluid-fluid levels, except for rare solid $A B C$ variants. Rarely, the $A B C$ is localized on the surface of the long bones [11]. Histopathologically, the lesions are composed of hemorrhagic tissue with cavitary spaces separated by fibrous septa composed of spindle cells, inflammatory cells and a smaller percentage of giant cells [12]. Treatment options are intralesional curettage followed by bone grafting, in combination with cryotherapy, sclerotherapy, radionuclide ablation, arterial embolization and en bloc resection [13,14]. Complications associated with curettage are related to an incomplete resectability of the lesion resulting in recurrence in at least 20\% [2]. Clinically [15], ABCs can be divided into inactive, active and aggressive lesions with aggressive tumors expanding rapidly, destroying surrounding

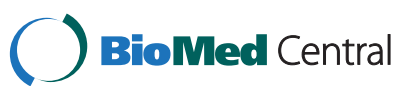

(c) 2014 Pauli et al.; licensee BioMed Central Ltd. This is an open access article distributed under the terms of the Creative Commons Attribution License (http://creativecommons.org/licenses/by/2.0), which permits unrestricted use, distribution, and reproduction in any medium, provided the original work is properly cited. 
tissue and having a high rate of local recurrences. New therapeutic options are needed for the management of this locally aggressive disease.

Denosumab, a monoclonal antibody specifically binding RANK-ligand, inhibits bone resorption and, therefore, [16-18] is used in the treatment of osteoporosis, skeletal complications of metastatic disease, and more recently in the treatment of giant cell tumors of bone, with a high rate of clinical success [19]. To date, we are aware of only one publication presenting the application of denosumab in two cases of spinal ABCs [20]. Both patients (an 8-year old boy and an 11-year old boy) recovered significantly from pain and neurological symptoms. MRI follow-up after two to four months of denosumab therapy showed tumor regression in both patients. We report a case of a locally aggressive periosteal $A B C$ with a confirmed rearrangement of USP6 arising in the radius of a 21-year old woman with an impressive local response to denosumab treatment and a follow-up of four years.

\section{Case presentation}

A 21-year old right-handed woman presented with a variable swelling and shooting pain in her right proximal forearm in May of 2009. Clinical examination showed a palpable swelling over the radial head mainly located over the biceps tendon and a supination deficiency. MRI revealed an extensive, deep seated, solid soft tissue tumor with contrast uptake, infiltration of the intra-osseous membranes, biceps tendon, contact with the neurovascular bundle, infiltration of the supinator muscle and deep extensor as well as deep flexor muscles (Figure 1A, B). Computer tomography (CT)guided core-needle biopsy was performed with a clinical suspicion of Ewing sarcoma. A low-grade, giant cell-containing lesion with focal metaplastic bone formation and infiltration of the skeletal muscle was diagnosed on histopathological examination (Figure 2A). No necrosis, atypia or pathologic mitotic activity was noted. The osteoclastic giant cells were numerous and contained up to over 50 nuclei.
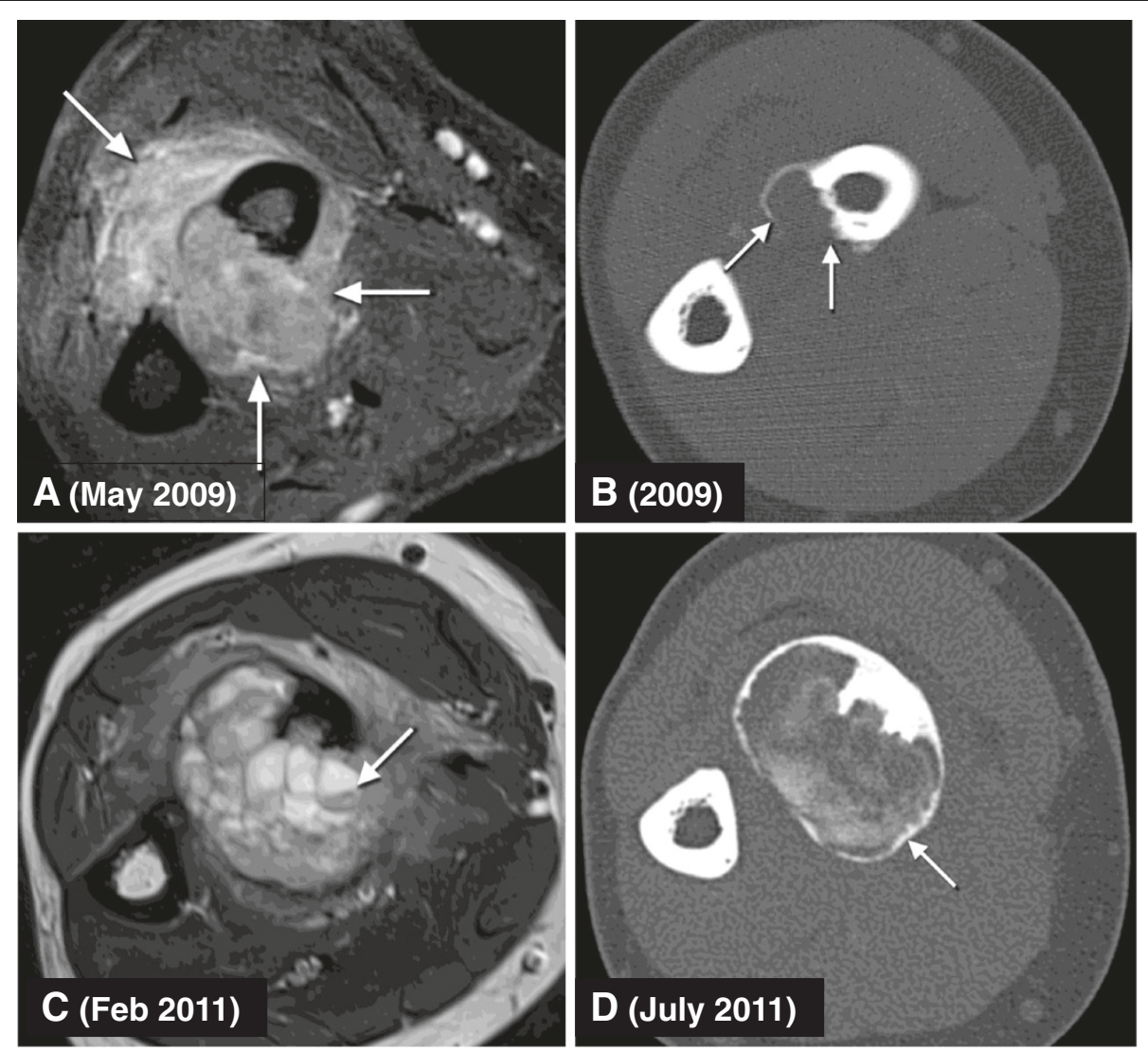

Figure 1 Imaging of the patients right forearm tumor. (A) Initial magnetic resonance imaging (MRI) demonstrating extensive involvement of the soft tissue between the radius and ulna as well as the cortex of the radius by an exclusively solid tumor mass (arrows). (B) Pre-treatment computer tomography (CT) scan with a small area of a split and disrupted cortex of the radius (arrows). (C) MRI directly prior to denosumab therapy with a locally progressive, extensive soft tissue mass following local surgical therapy 18 months previously. Fluid-fluid levels may be seen at this point (arrow). (D) CT scan following five months of denosumab therapy demonstrating almost complete containment of the soft tissue mass by a boney rim (arrow). 

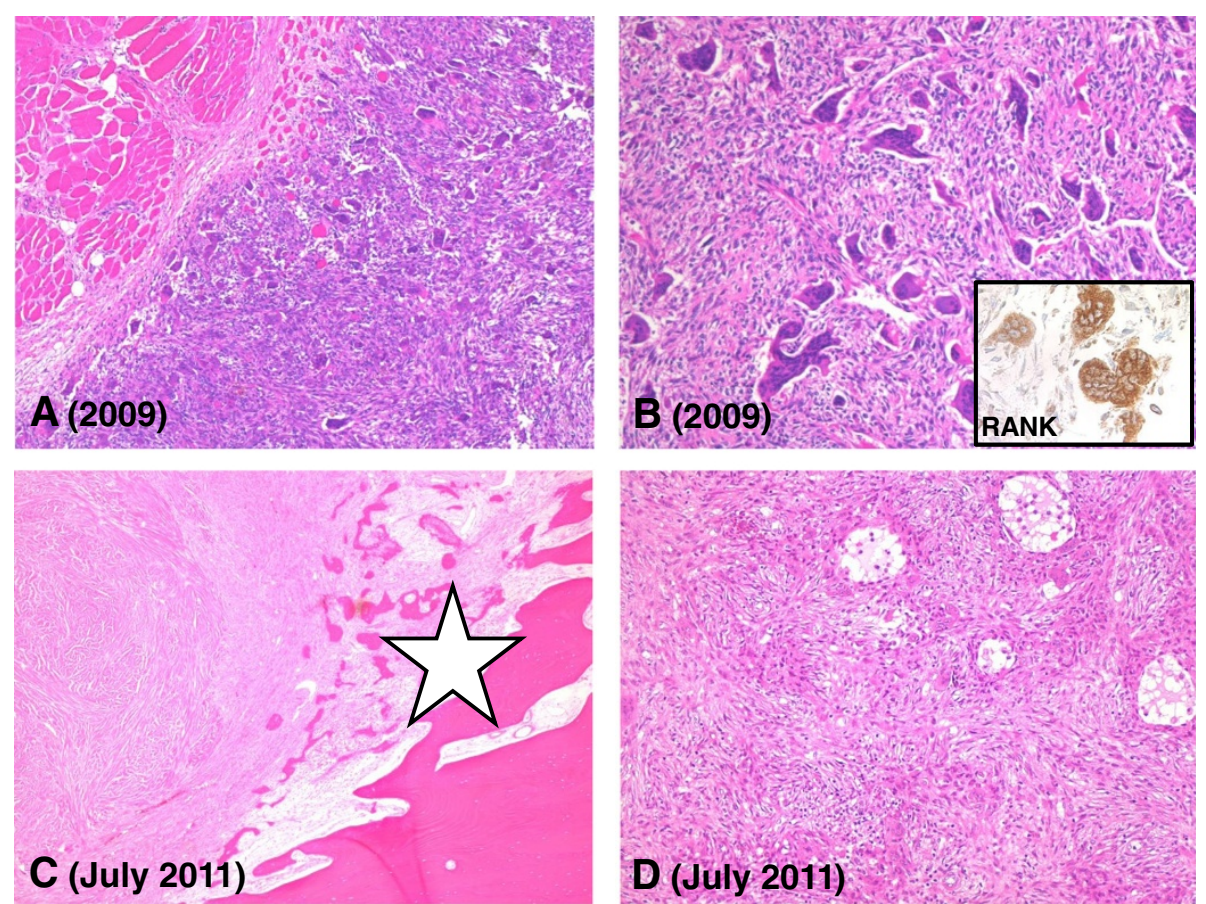

Figure 2 Histopathology of the pre- and post-treatment tumor tissue specimen. (A) Pre-treatment biopsy sample showing giant cell containing soft tissue mass with extensive infiltration of the skeletal muscle (H\&E stain; original magnification 50X). (B) Abundant lesional giant cells with numerous nuclei and mononuclear cells in the background (H\&E stain; original magnification 100X). Inset shows immunohistochemical expression of RANK (dilution 1:400; R\&D Systems, Abingdon, United Kingdom) by the osteoclastic giant cells. (C) Denosumab treatment induced boney containment (asterisk) of the tumor (upper left) (H\&E stain; original magnification 25X). (D) Post-denosumab-treatment tumor specimen showing pronounced reduction of the number of giant cells (H\&E stain; original magnification 100x).

Surgical therapy two months later consisted of a curettage through an antero-lateral and dorsal incision with dissection of the radial nerve branches to preserve maximal function. Histology of the recovered fragmented tumor revealed similar results to the biopsy (Figure 2B) rendering the diagnosis of an incompletely resected giant cell tumor of soft tissue. Residual tumor has been followed clinically and on imaging with a new local progression noticed at the end of 2010. MRI in February 2011 (Figure 1C) showed a significant increase in the size of a recurrent and progressively symptomatic tumor. Under the assumption of the diagnosis of a giant cell tumor of soft tissue, the therapy with denosumab $(120 \mathrm{mg}$ subcutaneously injected every month) for four months was administered with no untoward side effects. Followup MRI after one month of denosumab showed obvious tumor regression in size. Five months after the first denosumab injection, surgery was performed (July 2011). As a result of good treatment response, the tumor had become significantly better circumscribed and clearly demarcated by a bony rim (Figure 1D), leading to the feasibility of a complete resection of the tumor. Reconstruction was performed using an intercalary fibula-allograft with $3 \mathrm{D}$ planning to adjust for radial head rotation and a custom-made plate (Figure 3A). The gross examination of the resection specimen (Figure $3 \mathrm{~B}$ ) revealed an extensive, almost circumferential, intracortical bone tumor mass extending over $4.4 \mathrm{~cm}$ of the diaphysis of the radius with a small intramedullary nodule proximally and an intracortical satellite lesion distally. The histology of the post-treatment tumor specimen (Figure 2C, D) differed in comparison to the material of the core biopsy and curettage (Figure 2A, B) showing markedly reduced numbers of osteoclasts. The individual giant cells contained only few nuclei. Few small, pseudocystic spaces were observed in the background of collagen rich connective tissue, containing bland mononuclear cells. There was pronounced metaplastic new bone production with extensive areas of so-called 'blue bone' and lamellar cortical bone at the periphery of the lesion (asterisk Figure 2C). Due to the gross findings with almost exclusive intracortical tumor location, the lesion was reclassified as a predominantly intracortical, aneurysmal bone cyst of the proximal radius. Bone and soft tissue resection margins were free of tumor except for an unclear area at the distal corticalis.

At first presentation in 2009, most of the exclusively solid tumor mass was localized in the soft tissues of the 


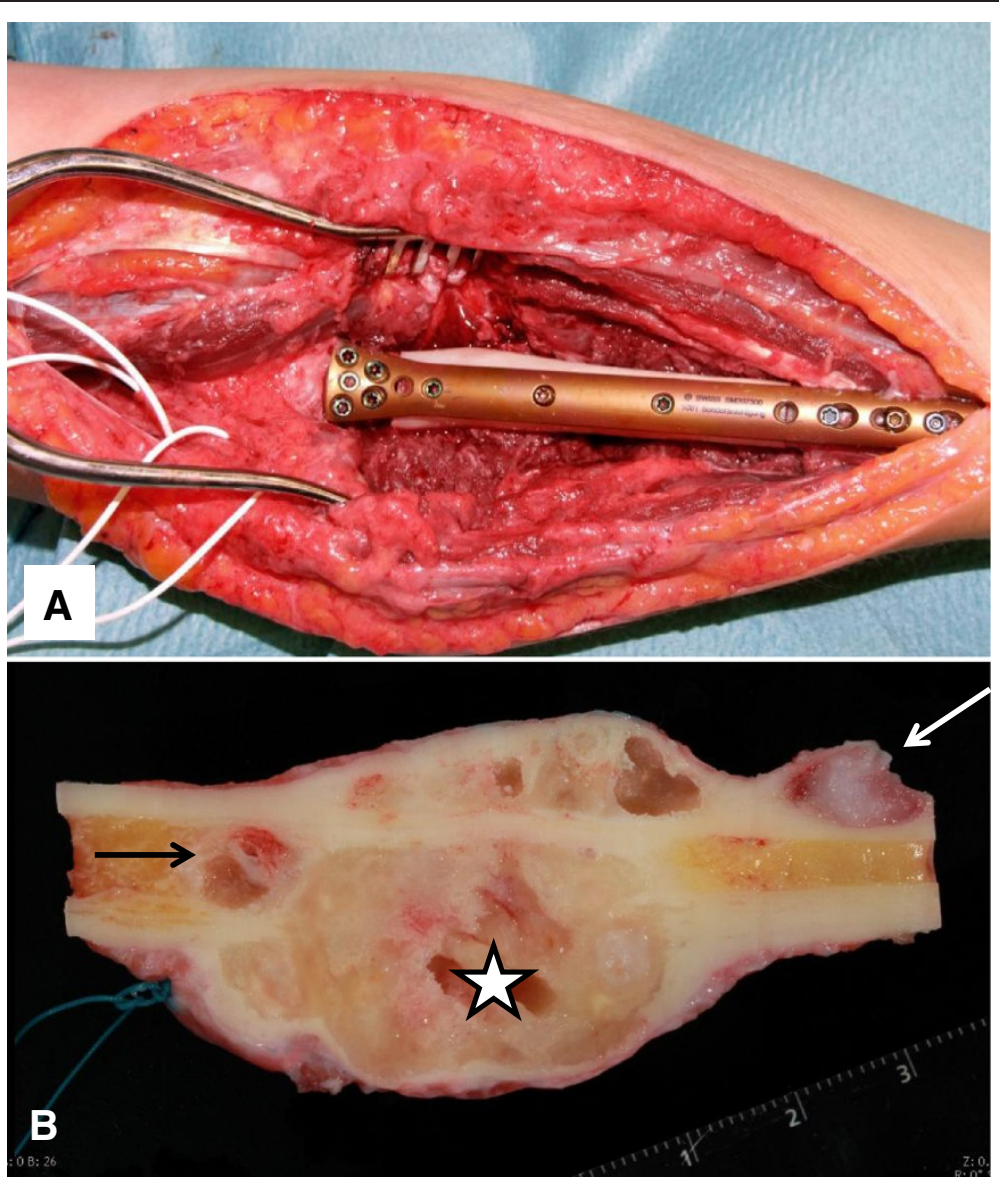

Figure 3 Resection of the shaft of the proximal radius following the denosumab therapy. (A) Reconstruction using an intercalary fibula-allograft and a custom-made plate. (B) Longitudinal section of the resected specimen demonstrating large intracortical, fully contained by a rim of bone tumor mass (asterisk) with a small intramedullar tumor nodule proximally (black arrow) and an intracortical satellite distally (white arrow).

forearm on imaging (Figure 1A), leading to the diagnosis of giant tumor of soft tissue. Retrospectively, careful analysis of the initial CT scans (Figure 1B) showed an area of split and disrupted cortical bone of the radius, which might have initially suggested the diagnosis of an intracortical (solid variant) ABC with a massive extension to the periosteal tissue. Finally, the diagnosis of a primary $\mathrm{ABC}$ was definitively confirmed by demonstration of a rearrangement of the USP6 gene locus by fluorescence in situ hybridization (FISH) as described previously [21], utilizing a custom-designed break apart probe set on the original biopsy sample (Figure 4). The mononuclear nuclei exhibit one fused red/green signal corresponding to a normal 17p USP6 locus (black arrow) and a pair of split green and red signals (white arrows) indicating a rearrangement of the USP6 locus consistent with the diagnosis of aneurysmal bone cyst.

Further follow up was unremarkable for another 19 months until a control MRI in February 2013 revealed a small $(<1 \mathrm{~cm})$ lesion in the area of the distal osteotomy and the core biopsy confirmed the local recurrence of the $A B C$, which is currently being treated with denosumab.

\section{Conclusion}

In this report, we describe a patient treated with denosumab for a locally aggressive, presumed giant cell tumor of soft tissue of the forearm. Evaluation of the resection specimen led to the final diagnosis of an intracortical aneurysmal bone cyst with massive infiltration of the soft tissue, a diagnosis which was also confirmed by FISH analysis.

Histopathologic differential diagnosis of osteoclastic giant cell containing lesions of skeletal tissues is extensive (giant cell tumor of bone, giant cell tumor of soft tissue, tenosynovial giant cell tumor, aneurysmal bone cyst, reparative giant cell granuloma, brown tumor of hyperparathyroidism, non-ossifying fibroma) and the final diagnosis has to be made in close conjunction 


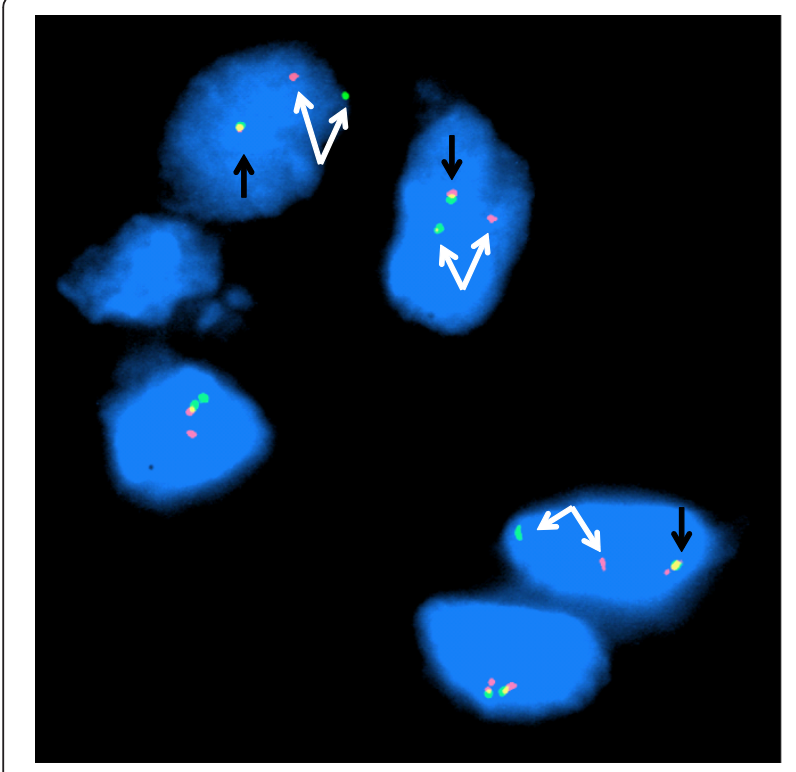

Figure 4 Fluorescence in situ hybridization conducted on the pretreatment specimen with a custom-designed break apart probe set; the probe cocktail proximal to the USP6 locus is labeled in green and distal in red. The mononuclear nuclei exhibit one fused red/green signal corresponding to a normal 17p USP6 locus (black arrow) and a pair of split green and red signals (white arrows) indicating a rearrangement of the USP6 locus consistent with the diagnosis of aneurysmal bone cyst. with clinical and radiological information. Giant cells occurring in both giant cell tumors (GCTs) and in $\mathrm{ABCs}$ are positive for markers of true osteoclasts, expressing RANK (receptor activator of NF-kB) and promoting bone destruction [22]. The formation of osteoclast type of giant cells in the GCTs is considered to be a consequence of the local production of RANKL (RANK-ligand) by the mononuclear, true neoplastic tumor cells [23]. A current therapeutic option for patients with giant cell tumors of bone is curettage with optimal preservation of function. However, patients undergoing curettage often have recurrent disease or significant morbidity. Denosumab is a human anti-RANKL monoclonal antibody that inhibits RANKL function and therefore its osteoclast activity, and causes the repression of osteoclast recruitment, maturation and bone resorption [16,18]. Denosumab is a Food and Drug Administration (FDA)-approved drug for osteoporosis and skeletal-related events in patients with bone metastases. A study to explore the action of denosumab in the treatment of giant cell tumor of bone was performed [19], demonstrating that the denosumab treated patients had a decrease in giant cells of 90 percent or greater, an indicator for the reduction of the aggressiveness of the tumors. The application of denosumab for bone tumors other than GCT has rarely been investigated. There exists only one report of denosumab use for $\mathrm{ABC}$ in two patients with spinal tumors [20]. However, the molecular confirmation of the primary $\mathrm{ABCs}$ as well as the histologic changes observed during and following therapy has not been previously reported.

Denosumab therapy was well tolerated by the current patient. The impressive positive treatment effects on the tumor tissue, as illustrated by marked reduction in the number of giant cells, were coupled with the bony demarcation of the tumor and facilitated subsequent function preserving surgery. The application of this form of the RANKL inhibition led to the transition of a locally aggressive and destructive tumor into a resectable lesion, thus preserving full function in our patient.

Our observations support the hypothesis that not only patients with GCTs, but also those suffering from ABCs may benefit substantially from RANKL-inhibiting denosumab treatment. These findings may offer new therapeutic options for often young patients with these locally aggressive tumors, especially in uncontrollable, locally destructive or recurrent disease.

\section{Consent}

Written informed consent was obtained from the patient for the publication of this report and any accompanying images.

\section{Abbreviations}

ABC: Aneurysmal bone cyst; CT: Computer tomography; FISH: Fluorescence in situ hybridization; GCT: Giant cell tumor; H\&E: Hematoxylin and eosin; MRI: Magnetic resonance imaging; RANK: Receptor activator of NF-K; RANKL: Receptor activator of NF-K ligand; USP6: Ubiquitin carboxyl-terminal hydrolase 6.

\section{Competing interests}

The authors indicate no potential conflict of interests.

\section{Authors' contributions}

$\mathrm{CP}$ and BB performed histopathologicals studies and drafted the manuscript. BF and $\mathrm{SH}$ coordinated the management and the treatment of the patient, concerning the surgeries and denosumab treatment respectively. CP coordinated the imaging studies. JB carried out the molecular genetic studies. All authors read and approved the final manuscript.

\section{Acknowledgments}

The authors would like to thank Dr. Yuanyuan Zhang at UNMC for her technical contributions.

\section{Author details}

${ }^{1}$ Institute of Surgical Pathology, University Hospital Zurich, CH-8091, Zurich, Switzerland. ${ }^{2}$ Orthopedics, University Hospital Balgrist, Zurich, Switzerland. ${ }^{3}$ Radiology, University Hospital Balgrist, Zurich, Switzerland. ${ }^{4}$ Department of Pathology/Microbiology, University of Nebraska Medical Center, Omaha, NE, USA. ${ }^{5}$ Oncology, University Hospital Zurich, Zurich, Switzerland.

Received: 23 August 2013 Accepted: 12 January 2014

Published: 20 January 2014 


\section{References}

1. Campanacci M, Cervellati C, Donati U, Bertoni F: Aneurysmal bone cyst (a study of 127 cases, 72 with long term follow up). Ital J Orthop Traumatol 1976, 2:341-353.

2. Mankin HJ, Hornicek FJ, Ortiz-Cruz E, Villafuerte J, Gebhardt MC: Aneurysmal bone cyst: a review of 150 patients. J Clin Oncol 2005, 23:6756-6762

3. Cottalorda J, Kohler R, Sales de Gauzy J, Chotel F, Mazda K, Lefort G, Louahem D, Bourelle S, Dimeglio A: Epidemiology of aneurysmal bone cyst in children: a multicenter study and literature review. J Pediatr Orthop B 2004, 13:389-394.

4. Lichtenstein L: Aneurysmal bone cyst; further observations. Cancer 1953, 6:1228-1237.

5. Oliveira AM, Hsi BL, Weremowicz S, Rosenberg AE, Dal Cin P, Joseph N, Bridge JA, Perez-Atayde AR, Fletcher JA: USP6 (Tre2) fusion oncogenes in aneurysmal bone cyst. Cancer Res 2004, 64:1920-1923.

6. Oliveira AM, Perez-Atayde AR, Inwards CY, Medeiros F, Derr V, Hsi BL, Gebhardt MC, Rosenberg AE, Fletcher JA: USP6 and CDH11 oncogenes identify the neoplastic cell in primary aneurysmal bone cysts and are absent in so-called secondary aneurysmal bone cysts. Am J Pathol 2004, 165:1773-1780.

7. Lau AW, Pringle LM, Quick L, Riquelme DN, Ye Y, Oliveira AM, Chou MM: TRE17/ubiquitin-specific protease 6 (USP6) oncogene translocated in aneurysmal bone cyst blocks osteoblastic maturation via an autocrine mechanism involving bone morphogenetic protein dysregulation. $J$ Biol Chem 2010, 285:37111-37120.

8. Oliveira AM, Chou MM, Perez-Atayde AR, Rosenberg AE: Aneurysmal bone cyst: a neoplasm driven by upregulation of the USP6 oncogene. J Clin Oncol 2006, 24:e1. author reply e2.

9. Ye Y, Pringle LM, Lau AW, Riquelme DN, Wang H, Jiang T, Lev D, Welman A, Blobel GA, Oliveira AM, Chou MM: TRE17/USP6 oncogene translocated in aneurysmal bone cyst induces matrix metalloproteinase production via activation of NF-kappaB. Oncogene 2010, 29:3619-3629.

10. Nielsen G, Fletcher C, Smith M: Soft tissue aneurysmal bone cyst: a clinicopathologic study of five cases. Am J Surg Pathol 2002, 26:64-69.

11. Maiya S, Davies M, Evans N, Grimer J: Surface aneurysmal bone cysts: a pictorial review. Eur Radiol 2002, 12:99-108.

12. Dabska MBJ: Aneurysmal bone cyst: pathology, clinical course and radiologic appearances. Cancer 1969, 23:371-389.

13. Garg SMS, Dormans JP: Modern surgical treatment of primary aneurysmal bone cyst of the spine in children and adolescents. J Pediatr Orthop 2005, 25:387-392.

14. Peeters SP, Van der Geest IC, de Rooy JW, Veth RP, Schreuder HW: Aneurysmal bone cyst: the role of cryosurgery as local adjuvant treatment. J Surg Oncol 2009, 100:719-724.

15. Enneking WF: A system of staging musculoskeletal neoplasms. Clin Orthop Relat Res 1986, 204:9-24.

16. Hanley DA, Adachi JD, Bell A, Brown V: Denosumab: mechanism of action and clinical outcomes. Int J Clin Pract 2012, 66:1139-1146.

17. Lipton A, Jacobs I: Denosumab: benefits of RANK ligand inhibition in cancer patients. Curr Opin Support Palliat Care 2011, 5:258-264.

18. Moen MD, Keam SJ: Denosumab: a review of its use in the treatment of postmenopausal osteoporosis. Drugs Aging 2011, 28:63-82.

19. Thomas D, Henshaw R, Skubitz K, Chawla S, Staddon A, Blay J-Y, Roudier M, Smith J, Ye Z, Sohn W, et al: Denosumab in patients with giant-cell tumor of bone: an open-label, phase 2 study. Lancet Oncol 2010, 11:275-280.

20. Lange $T$, Stehling $C$, Frohlich $B$, Klingenhofer M, Kunkel P, Schneppenheim R, Escherich G, Gosheger G, Hardes J, Jurgens H, Schulte TL: Denosumab: a potential new and innovative treatment option for aneurysmal bone cysts. Eur Spine J 2013, 22:1417-1422.

21. Althof PA, Ohmori K, Zhou M, Bailey JM, Bridge RS, Nelson M, Neff JR, Bridge JA: Cytogenetic and molecular cytogenetic findings in 43 aneurysmal bone cysts: aberrations of $17 p$ mapped to 17p13.2 by fluorescence in situ hybridization. Mod Pathol 2004 17:518-525

22. Won K, Kalil R, Kim Y, Park Y-K: RANK signaling in bone lesions with osteoclast-like giant cells. Pathology 2011, 43:318-321.

23. Thomas D: RANKL, denosumab, and giant cell tumor of bone. Curr Opin Oncol 2012, 24:397-403.

doi:10.1186/1477-7819-12-17

Cite this article as: Pauli et al:: Response of an aggressive periosteal aneurysmal bone cyst (ABC) of the radius to denosumab therapy. World Journal of Surgical Oncology 2014 12:17.

\section{Submit your next manuscript to BioMed Central and take full advantage of:}

- Convenient online submission

- Thorough peer review

- No space constraints or color figure charges

- Immediate publication on acceptance

- Inclusion in PubMed, CAS, Scopus and Google Scholar

- Research which is freely available for redistribution

Submit your manuscript at www.biomedcentral.com/submit
Biomed Central 